\title{
Preliminary Experience With the Use of Electromagnetic Navigation for the Diagnosis of Peripheral Pulmonary Nodules and Enlarged Mediastinal Lymph Nodes
}

\author{
Luis M. Seijo, ${ }^{\mathrm{a}}$ Gorka Bastarrika, ${ }^{\mathrm{b}}$ María Dolores Lozano, ${ }^{\mathrm{c}}$ and Javier J. Zulueta ${ }^{\mathrm{a}}$ \\ aDepartamento de Neumología, Clínica Universitaria de Navarra, Universidad de Navarra, Pamplona, Navarra, Spain \\ bDepartamento de Radiología, Clínica Universitaria de Navarra, Universidad de Navarra, Pamplona, Navarra, Spain \\ 'Departamento de Anatomía Patológica, Clínica Universitaria de Navarra, Universidad de Navarra, Pamplona, Navarra, Spain
}

Electromagnetic navigation is a new technique that can be used with bronchoscopy to obtain samples of small peripheral nodular lesions and enlarged mediastinal lymph nodes. It is very versatile in that it enables both transbronchial biopsies and fine-needle aspiration to be performed. We describe 2 cases in which navigation with the superDimension/ Bronchus system combined with traditional diagnostic techniques facilitated a definitive diagnosis by bronchoscopy. Electromagnetic navigation can obviate the need for more invasive diagnostic procedures such as surgery, thus saving time and money and avoiding complications.

Key words: Bronchoscopy. Navigation. Biopsy.
La navegación electromagnética en el diagnóstico de nódulos periféricos y adenopatías

mediastínicas: experiencia preliminar

La navegación electromagnética es una técnica novedosa capaz de facilitar la obtención mediante broncoscopio de muestras de lesiones nodulares periféricas de pequeño tamaño y adenopatías mediastínicas. Permite realizar tanto biospias transbronquiales como punciones con aguja citológica, y por ello es muy versátil. Publicamos el resultado de $\mathbf{2}$ casos en los que la combinación de navegación con el sistema superDimension/Bronchus y de técnicas diagnósticas convencionales facilitó el diagnóstico definitivo mediante broncoscopia. La navegación electromagnética ofrece la posibilidad de evitar métodos diagnósticos invasivos como la cirugía, con el consiguiente ahorro económico, de tiempo y de complicaciones.

Palabras clave: Broncoscopia. Navegación. Biopsia.

\section{Introduction}

Peripheral pulmonary nodules remain a challenge for the bronchoscopist. The diagnostic yield of transbronchial biopsy is very limited in this case and is no more than $15 \%$ to $30 \%$ in lesions under $2 \mathrm{~cm}$, especially if these are in the distal third of the airway. ${ }^{1}$ The same could be said about fine needle aspiration of enlarged mediastinal lymph nodes, albeit for different reasons. In this case, the frustration lies in the difficulty in reproducing the diagnostic yield of very experienced hospitals, ${ }^{2}$ especially those, such as ours, that are fortunate enough to have a histopathologist present during the procedure.

\footnotetext{
Correspondence: Dr. L.M. Seijo.
}

Departamento de Neumología. Clínica Universitaria de Navarra.

Avda. Pío XII, 36. 31008 Pamplona. Navarra. España.

E-mail:1mseijo@unav.es

Manuscript received October 31, 2006

Accepted for publication December 12, 2006.
None of the techniques developed to date, including biopsy guided by endoscopy or computed tomography (CT), ${ }^{3}$ have significantly increased the diagnostic yield as they are all limited by the difficulty in reaching a peripheral lesion with a rigid instrument. In general, the smaller the lesion, the lower the diagnostic yield. Only endoscopic ultrasound seems able to guarantee the success of mediastinal transbronchial fine needle aspiration, ${ }^{4,5}$ since it provides real-time observation of the enlarged lymph nodes to be aspirated. However, it is very expensive and the technique takes a long time to learn.

Electromagnetic navigation enables us to carry out a highly accurate biopsy of small peripheral nodules and enlarged mediastinal lymph nodes using conventional bronchoscopy. This novel technique is successful for 3 reasons: a) prior virtual planning with a multislice CT, b) real-time navigation, and c) a flexible electromagnetic probe that can negotiate the turns and bifurcations of the airway to reach a remote lesion. 


\section{Methods}

\section{Description of the Technique}

The Bronchus system (superDimension Europe, Düsseldorf, Germany) is composed of 4 basic elements: $a$ ) an electromagnetic field surrounding the thorax; $b$ ) a manually assembled and calibrated steerable electromagnetic probe; $c$ ) an extended working channel reinforced with titanium at the distal end to enable biopsy material to be obtained easily; and $d$ ) the necessary software to plan the intervention and provide a real-time visual guide indicating the location of the probe. All the interventions are made using a flexible videobronchoscope (the working channel must be $2.8 \mathrm{~mm}$ to enable the extended channel to be inserted) under sedation or, in some cases, general anesthesia. In our hospital, we use a combination of midazolam and fentanyl or propofol with topical anesthetic.

All patients have to undergo a CT wit 2- to $3 \mathrm{~mm}$ slices and 1- to 1.5- mm intervals in DICOM format. The CT data are recorded on a compact disc, thereby enabling the intervention to be planned using a virtual reconstruction of the bronchial tree.

\section{Registration, Navigation, and Sampling}

Reconstruction of the bronchial tree allows virtual reference points to be marked. These points, such as the main carina, are generally easily accessed and allow the triangulation system to position the probe within the electromagnetic field and, therefore, within the thorax during the intervention. Similarly, one or more targets (a pulmonary nodule and/or enlarged mediastinal lymph node) and, occasionally, several intermediate reference points (eg, subsegmental carinae) are marked, thus facilitating systematic exploration of the airway until the target is reached. During bronchoscopy, the extended working channel and the probe are introduced into the bronchial tree and the reference points selected during the planning stage are registered. This process is carried out by touching each reference point (eg, the main carina) gently with the distal end of the probe and pressing a pedal. The software then compares the 2 images - virtual and real-and indicates the degree of divergence between them (eg, virtual main carina versus actual main carina), which, in optimal conditions, should not be more than $4 \mathrm{~mm}$. Once registration has been completed, the navigation process can start. During the process, the operator can always see a 3-dimensional reconstruction on the scanner. The position of the probe in the virtual scenario depends on its position within the electromagnetic field. Correct functioning of the system is easily verified by placing the probe at any visible point in the bronchial tree and confirming that the location of the probe in the virtual scenario corresponds with its actual position (eg, a segmental bronchus in the right lower lobe). Navigation is carried out by simultaneously advancing the working channel and the probe gently until the lesion is reached. The system enables the operator to verify that the probe is approaching the lesion or whether, on the contrary, it is moving away from it. If this is the case, the operator can redirect the probe using the 3 projections as a guide and rectifying until the bronchus containing the target is reached. Navigation to a mediastinal lymph node is much simpler, as there is no need to enter the bronchial tree. In both cases, the software calculates the distance and direction to the center of the target by taking the tip of the probe as a reference.

Once the nodule or lymph node has been reached, the working channel is fixed and the probe is withdrawn. The biopsy forceps or needle is then introduced and the samples obtained. In our hospital, a histopathologist is present to ensure the success of the procedure and to check that the material extracted is suitable for diagnosis. Some hospitals visualize the biopsy procedure using a fluoroscope, although we think that this is not necessary.

\section{Indications}

Electromagnetic navigation is indicated for the diagnosis of all types of pulmonary nodules and enlarged mediastinal lymph nodes. It is particularly useful when a nodule is small $(<2.5 \mathrm{~cm})$ or difficult to reach using CT-guided aspiration. It is also useful in the aspiration of small lymph nodes or those from which it is traditionally difficult to obtain samples, such as the hilar or upper paratracheal lymph nodes.

\section{Limitations}

The system is limited by the size of the target nodule to be biopsied, which is restricted to approximately $8 \mathrm{~mm}$ to $10 \mathrm{~mm}$. Some lesions, such as those found in the upper lobes, are more difficult to reach. Logically, the technique requires a learning period-approximately 10 interventions-and both additional cost and prolongation of the intervention, generally by no more than 10 minutes. The system is also limited by the need to use a bronchoscope with a $2.8-\mathrm{mm}$ working channel, the fact that secretions and blood cannot be aspirated during navigation, and the need for a scanner with specific slices and overlap in order to maximize planning. Lastly, some nodules (probably about $10 \%$ ) cannot be reached due to their position or remoteness.

\section{Contraindications}

The contraindications are the same as for any bronchoscopy. In addition, there is a hypothetical risk of interference between the electromagnetic field and devices such as pacemakers and defibrillators, although the magnitude of this field is lower than that of the earth.

\section{Case Description}

\section{Peripheral Nodule}

A 45-year-old man, ex-smoker, with no clinical history of interest, consulted for evaluation of a pulmonary mass. He began to have frank hemoptysis in April 2006. A chest $x$-ray showed a lesion of the right upper lobe. The CT scan confirmed the presence of a spiculated cavitated 2-cm nodule in this area; both bronchoscopy and CT-guided aspiration were performed without success. As diagnosis was uncertain, the patient's physicians proposed surgery. The patient refused and remained asymptomatic until July when the hemoptysis returned. He came to our hospital complaining of vague discomfort in the shoulder and right hemithorax, accompanied by chronic morning expectoration, with no fever, weight loss, or dyspnea. The physical examination was normal. A repeat bronchoscopy using electromagnetic navigation was proposed, with a prior planning CT scan (Figure 1). Three biopsies and several aspirates were obtained after navigating with the electromagnetic probe to a distance of $5 \mathrm{~mm}$ from the nodule. The histopathologist, who was present during the examination, confirmed the presence of extensive inflammation; therefore, material from 1 of the aspirations was sent to the microbiology laboratory. The culture revealed Prevotella melaninogenica, Peptostreptococcus micros, and Streptococcus salivarius, and the biopsy report showed chronic inflammation. A course of antibiotics (Augmentin) was started and surgery was rejected. The nodule disappeared with the antibiotic prescribed. 

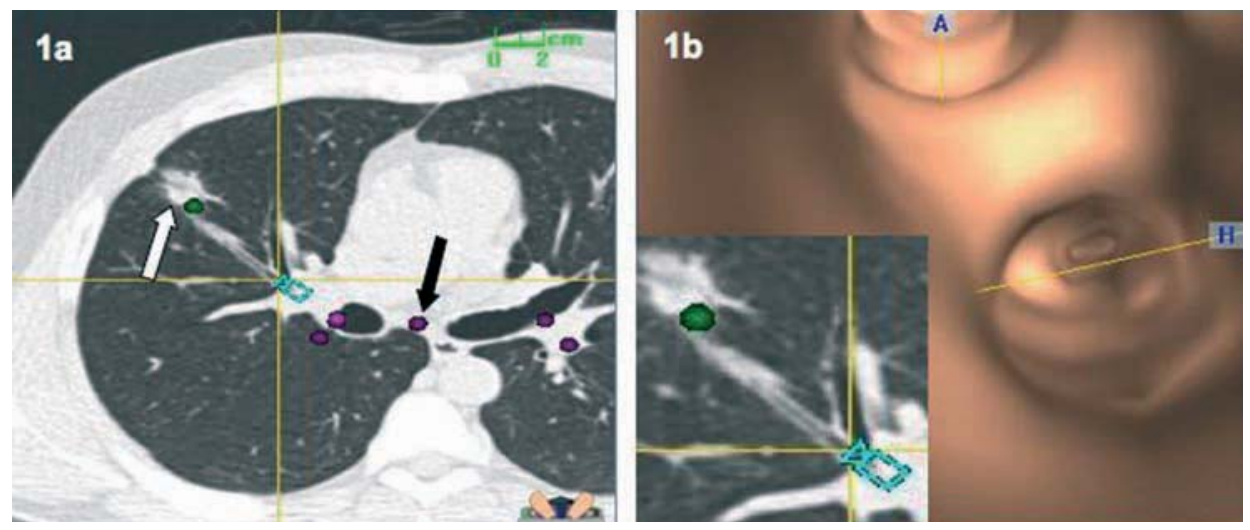

Figure 1. Prior planning (a) allows reference points such as the main carina (black arrow) and the target nodule (white arrow) to be marked, thus facilitating highly accurate navigation. Virtual reconstruction of the bronchial tree $(b)$ identifies the bronchus that leads to the lesion. Biopsies, aspirates, and cultures were obtained, and all indicated a diagnosis of pulmonary abscess.

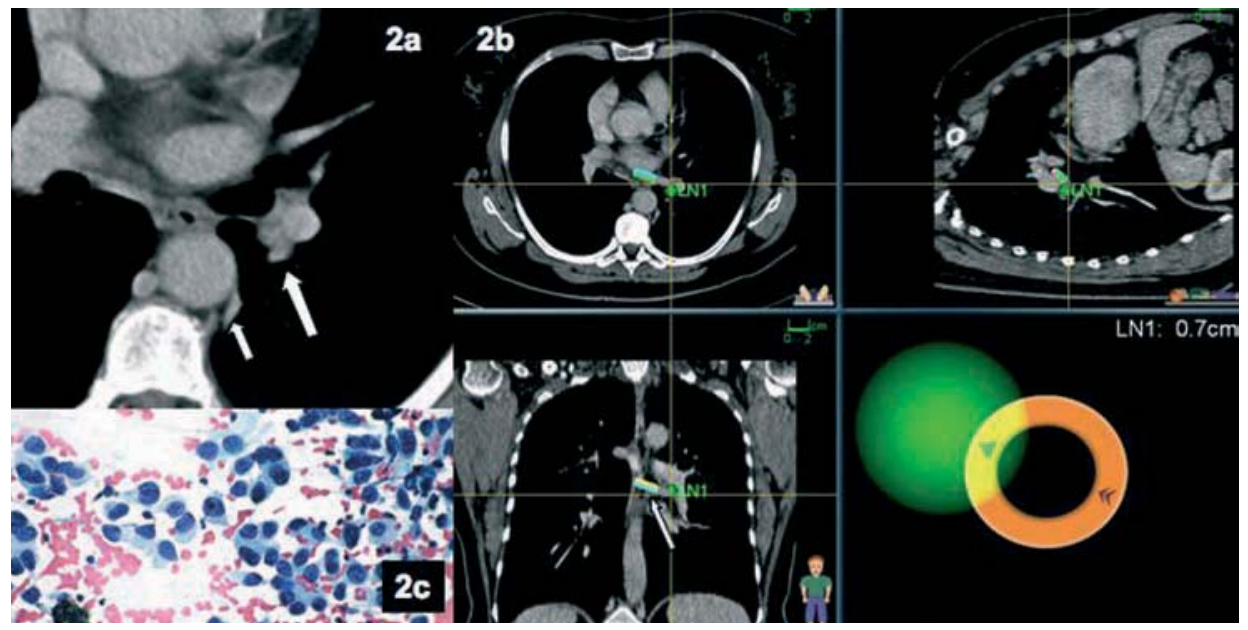

Figure 2. Conventional computed tomography scan $(a)$ identifying the presence of an inaccessible paraaortic pulmonary nodule (small arrow) and slight thickening of the left hilum (large arrow) with intense uptake in positron emission tomography. Image of the navigation $(b)$ with the probe (arrow) at $0.7 \mathrm{~cm}$ from the lymph node, allowing transbronchial aspiration to be performed. The histopathologist, who was present during the intervention, confirmed that this was an adenocarcinoma $(c)$.

\section{Diseased Hilar Lymph Node}

A 52-year-old woman, nonsmoker, consulted for evaluation of a pulmonary nodule. She had recently been diagnosed with seronegative rheumatoid arthritis and presented von Willebrand disease. Due to her persistent dyspnea and cough, a noncontrast CT scan of the chest was performed. The scan revealed a small, para-aortic lesion $(1 \mathrm{~cm})$ with no visible enlarged lymph nodes (Figure 2). Positron emission tomography was performed, revealing uptake not only in the known pulmonary nodule, but also in the left hilar region. Therefore, a diagnostic bronchoscopy with electromagnetic navigation was proposed, with prior prophylactic administration of desmopressin. Three fine-needle aspirates were taken and the histopathologist was present during the examination (Figure 2). An adenocarcinoma of possible pulmonary origin was diagnosed.

\section{Discussion}

The superDimension electromagnetic navigation system enables us to obtain biopsies of enlarged mediastinal lymph nodes and small peripheral lung nodules. Its reliability and accuracy were recently demonstrated in a trial carried out by the Cleveland Clinic. ${ }^{6}$ The mean size of the biopsied nodules in that study was $22.8 \mathrm{~mm}$, and of the lymph nodes $28.1 \mathrm{~mm}$, and the diagnostic yield of bronchoscopy was $74 \%$ and $100 \%$, respectively. The only complications were
2 cases of pneumothorax in a total of 60 patients, and the navigation times were quite short, with a mean of 7 and 2 minutes, respectively. At our hospital, we have carried out 8 interventions during a short period, of which 6 were diagnostic ( 1 carcinoid, 1 abscess, 1 case of tuberculosis, 2 adenocarcinomas, and 1 squamous cell carcinoma). The mean size of the biopsied nodules at our hospital was $2.5 \mathrm{~cm}$ and there were no cases of pneumothorax. A report on our experience has been sent to the Spanish Society of Pulmonology and Thoracic Surgery (SEPAR).

Preintervention planning, which is usually carried out using a laptop computer, is essential if the length of the procedure is to be reduced. The probe is steerable, allowing it to negotiate the turns and bifurcations of the airway until it reaches the target node. This is impossible with rigid instruments such as forceps or a fine needle. Lastly, the presence of a histopathologist during the examination allows the diagnosis to be confirmed in situ, ensuring the success of the intervention. We think that the Bronchus system can be particularly useful in hospitals that do not have this service, since it enables mediastinal needle aspiration to be performed as reliably and accurately as with endoscopic-ultrasound guidance.

Despite its limitations, this versatile system promises to revolutionize the management of solitary pulmonary nodules and involve the bronchoscopist not just in making 


\section{SEIJO LM ET AL. PRELIMINARY EXPERIENCE WITH THE USE OF ELECTROMAGNETIC NAVIGATION FOR THE DIAGNOSIS OF PERIPHERAL PULMONARY NODULES AND ENLARGED MEDIASTINAL LYMPH NODES}

decisions but, also in obtaining diagnostic material. The cost of the system and the individual probes seems quite acceptable, ${ }^{7}$ since this technique avoids the need for much more expensive diagnostic surgery.

\section{REFERENCES}

1. Baaklini WA, Reinoso MA, Gorin AB, Sharafkaneh A, Manian P. Diagnostic yield of fiberoptic bronchoscopy in evaluating solitary pulmonary nodules. Chest. 2000;117:1049-54.

2. Horrow EM, Wajdy A, Blum J, Harkin T, Gasparini S, AddrizzoHarris DJ, et al. The utility of transbronchial needle aspiration in the staging of bronchogenic carcinoma. Am J Respir Crit Care Med. 2000;161:601-7.
3. Shinagawa N, Yamazaki K, Onodera Y, Miyasaka K, Kikuchi E, Dosaka Akita H, et al. CT-guided transbronchial biopsy using an ultrathin bronchoscope with virtual bronchoscopic navigation. Chest. 2004;125:1138-43.

4. Yasufuku K, Chiyo M, Sekine Y, Chajed P, Shibuya K, Iizasa T, et al. Realtime endobronchial ultrasound-guided transbronchial needle aspiration of mediastinal and hilar lymph nodes. Chest. 2004;126:1228.

5. Herth F, Becker HD, Ernst A. Conventional vs endobronchial ultrasound guided transbronchial needle aspiration: a randomized trial. Chest. 2004;125:322-5.

6. Gildea TR, Mazzone PJ, Karnak D, Meziane M, Mehta A. Electromagnetic navigation diagnostic bronchoscopy. Am J Respir Crit Care Med. 2006:174:982-9.

7. Schwarz Y, Greif J, Becker HD, Ernst A, Mehta A. Real-time electromagnetic navigation bronchoscopy to peripheral lung lesions using overlaid CT images. Chest. 2006;129:988-94. 